\title{
Caracterización de Arenas del Procesamiento de Caolín
}

\author{
Dulce Y. Palafox-Manríquez a,1 , Diana L. Vázquez-Martínez a,2, José E. Domínguez-Bravo a,3, Gerardo Cruz-López a,4, Felipe \\ Legorreta-García a, 5, *, Leticia E. Hernández-Cruz ${ }^{\text {a, }} 78$. \\ a 1 Universidad Autónoma del Estado de Hidalgo, Área Académica de Ciencias de la Tierra y Materiales, Carr. Pachuca-Tulancingo km 4.5 s/n, Mineral de la \\ Reforma, Hidalgo, México.
}

\begin{abstract}
Resumen
Arenas provenientes del procesamiento de caolín son caracterizadas de manera exhaustiva. Se realizó un análisis cristalográfico por medio de difracción de rayos X, análisis de la distribución de tamaño de partículas por tamizado, así como por difracción láser; se determinó la humedad, la densidad, el porcentaje de material magnético y la morfología. Los estudios cristalográficos ponen en evidencia que las fases que más predominan son sílice, caolinita, anortoclasa y alunita. Se encontró un tamaño promedio de partícula global de $46.34 \mu \mathrm{m}$. Las imágenes de microscopía óptica ponen en evidencia diferentes tipos de partícula de diferentes tonalidades correspondiendo a cuarzo polimórfico, silicoaluminatos y feldespatos.
\end{abstract}

Palabras Clave: Arenas de Caolín, caracterización, magnetismo.

\section{Introducción}

Frente a las necesidades del abastecimiento de materias primas para distintas industrias tales como la farmacéutica, la del papel, pinturas entre otras, es necesario el consumo del caolín sin embargo, la necesidad de obtenerlo con una calidad aceptable, produce desecho de material inorgánico cuya calidad es inferior. Tal es el caso de las arenas que proceden del desecho del procesamiento del caolín cuyo porcentaje en masa puede llegar a ser del $50 \%$ [1]. Es por esta causa que se buscan opciones que permitan reciclar este material, motivo por el cual se vislumbra la necesidad de realizar su caracterización física y química. Se ha documentado, en diferentes publicaciones referentes al empleo de residuo de caolín, que contiene un porcentaje de alúmina que oscila de un 20 a un $40 \%$. Se ha propuesto emplearse para la producción de cerámicos refractarios, cementos, block para construcción, vidrios cerámicos, síntesis de zeolita, entre otros [2-8]. El residuo de la industria del procesamiento de caolín es también llamado "kira" por diferentes autores. Se han propuesto varios procesos a fin de reciclar las arenas de caolín, por ejemplo, han sido mezclados con calcita, dolomita y ceniza y residuo de la industria del papel para producir cerámicas refractarias [3]. Rodríguez Meneses et al., han mezclado el residuo de caolín con alúmina para la fabricación de mullita; los autores demuestran que una gran cantidad de residuo presenta una fase de vidrio formada durante el sinterizado que perjudica al sistema. En otro trabajo del mismo autor, produce una mezcla de residuos del procesamiento de caolín, con ball clay y alúmina, para ser empleados en la elaboración de mullita. Los autores realizaron además un diseño estadístico, con la finalidad de encontrar las mezclas adecuadas y temperatura que generen propiedades mecánicas apropiadas [3-5]. La mezcla de arena de caolín con otros materiales naturales ha sido estudiado por Toya et al. quienes mezclaron el residuo

1 Dulce Yamel Palafox Manríquez.-Estudiante del segundo semestre del Programa Educativo de Ingeniería de Materiales de la UAEH

2 Diana Laura Vázquez Martínez.- Estudiante del segundo semestre del Programa Educativo de Ingeniería de Materiales de la UAEH

3 José Emilio Domínguez Bravo.- Estudiante del segundo semestre del Programa Educativo de Ingeniería de Materiales de la UAEH

4 Gerardo Cruz López.- Estudiante del segundo semestre del Programa Educativo de Ingeniería de Materiales de la UAEH

5 Felipe Legorreta García.- Profesor Investigador Titular Tiempo Completo. Doctor en Ciencias con Especialidad en Ciencia e Ingeniería de Materiales

(Science et Génie des Matériaux) por la Universidad Paul Sabatier Toulouse - Francia Nivel SIN 1.

6 Leticia E. Hernández Cruz.- Profesora Investigadora Titular Tiempo Completo. Doctora en Ciencias con especialidad en Metalurgia y Materiales por el

Instituto Politécnico Nacional.; Nivel SNI 1.

*Autor de Correspondencia

Correos electrónicos: dush2205@gmail.com (primer autor),anaid_urual@hotmail.com (segundo autor), joshemilio1998@gmail.com (tercer autor), gerardocr015@gmail.com (cuarto autor), felegorreta@hotmail.com (quinto autor) lesperanza.hernandez@ gmail.com (sexto autor). 
referido con dolomita $\left(\mathrm{CaMgCO}_{3}\right)$, con la finalidad de elaborar vidrio cerámico, por el método de reacción en estado sólido, o bien para la síntesis de cordierita [6, 7]. Por otro lado Azeredo et al., desarrollaron un concreto autocompacto con adición de residuos de la fabricación de caolín, los autores refieren que encontrando que este material a una dosificación adecuada, es factible obtener una mezcla superplastificante compatible con los materiales de concreto [8]. También el residuo de caolín de bajo porcentaje de alúmina ha sido empleado para la fabricación de block de construcción por Gómez et al., el residuo es activado con silicato de sodio e hidróxido de calcio [9]. Los resultados del trabajo mencionado ponen en evidencia que la activación promueve la formación de geo polímeros, y que la adición de silicato de sodio mejora las propiedades mecánicas del block fabricado. En otros trabajos, se ha obtenido la síntesis de zeolitas a partir de residuos de caolín. Maia et al., elaboran la zeolita tipo A [10] y por Hildebrando et al. [11], quienes sintetizan la zeolita tipo $\mathrm{NaP}$, ambas síntesis tratan el residuo con $\mathrm{NaOH}$ por el método hidrotermal. Sin embargo, los investigadores primeramente calcinan el residuo de caolín con la finalidad de activarlo. En este trabajo de investigación el residuo de caolín es caracterizado de manera exhaustiva por diferentes técnicas de análisis.

\section{Materiales y Métodos}

Las arenas, residuos de la planta piloto desarrollada en el Laboratorio de Tecnología de Cerámicos de la UAEH; se secaron en una estufa marca Thermo Cientific a una temperatura de $100^{\circ} \mathrm{C}$ durante 24 horas. El material fue homogeneizado y cuarteado hasta obtener 12 muestras representativas de $200 \mathrm{~g}$ cada una. A fin de efectuar el análisis por mallas, fue necesario someter una muestra al proceso de atrición ya descrito en un trabajo anterior [12], para el cual, $200 \mathrm{~g}$ de muestra, $400 \mathrm{ml}$ de agua y $0.5 \mathrm{ml}$ de silicato de sodio (empleado como dispersante), se atricionaron en una celda de flotación tipo DENVER a $3000 \mathrm{rpm}$, durante 7 minutos. Posteriormente la pulpa atricionada se vertió sobre las mallas \#400, \#325 ,\#200, \#150, \#120, \#100, \#80, \#50 Standard Test Sieve W.S. TYLER U.S.A., agregándole agua hasta observar que el agua fuese translucida y se secó el producto de cada malla, para su posterior análisis. Los polvos fueron analizados por DRX en el equipo Inel Equinox 2000, en un intervalo de $10^{\circ}$ a $80^{\circ}$ utilizando radiación de Cobalto (CoK $\left.\alpha 1\right)$. Se realizó además un análisis de óxidos por espectroscopía de energía dispersiva EDS del inglés (Electronic Dispersive Spectrometer), equipo acoplado a un microscopio electrónico modelo JEOL modelo 6300. Se determinó el tamaño por medio de un analizador de tamaño de partícula de difracción láser marca Beckman \& Coulter, modelo LS-13320 únicamente para las partículas más finas, es decir las que pasaron por la \#400. La densidad de la arena seca, se determinó con un matraz de Le Chatelier, el procedimiento se realizó cinco veces y se calculó el promedio. También se determinó el porcentaje de material magnético por medio de dos maneras la primera fue con el tubo Davis de laboratorio a 8 movimientos por minuto aplicando el máximo amperaje y la segunda simplemente acercando un imán de neodimio-hierroboro. Con la finalidad de determinar la morfología, color y confirmar el tamaño promedio de cada muestra, se empleó un Microscopio de tipo óptico USB de la marca "Comet".
Finalmente las pérdidas de masa por ignición (P.P.I), fueron determinadas sometiendo la muestra a calcinación a $800^{\circ} \mathrm{C}$, durante una hora, midiendo la masa inicial y final.

\section{Resultados y discusión}

\subsection{Análisis Químico}

La tabla del análisis químico obtenidos por medio de EDS, son mostrados en la Tabla 1. Se aprecia claramente que la mayor proporción corresponde a la sílice, conteniendo $54.88 \%$ en masa y en segundo lugar se encuentra la alúmina con $19.57 \%$. $\mathrm{Se}$ encontró además la presencia de otros óxidos que $\mathrm{Fe}, \mathrm{Na}$, $\mathrm{K}$, Ti y Ca, comúnmente encontrado en las arcillas de origen caolinítico - feldespático. La pérdida por ignición (P.P.I.), es también es presentado, es uno de los valores más altos debido a la demisión del agua que se encuentra en la estructura, carbonatos, sulfuros, material orgánico y otros materiales que se descomponen a $800^{\circ} \mathrm{C}$.

Tabla 1. Análisis químico de óxidos por espectroscopía de energía dispersiva EDS de la arena de caolín. (P.P.I. Pérdida por ignición).

\begin{tabular}{lllllllll}
\hline FASE & $\mathrm{SiO}_{2}$ & $\mathrm{Al}_{2} \mathrm{O}_{3}$ & $\mathrm{Fe}_{2} \mathrm{O}_{3}$ & $\mathrm{Na}_{2} \mathrm{O}$ & $\mathrm{K}_{2} \mathrm{O}$ & $\mathrm{TiO}_{2}$ & $\mathrm{CaO}$ & P.P.I \\
\hline \% EN & 54.88 & 19.57 & 4.40 & 2.04 & 0.78 & 2.19 & 0.38 & 15.76 \\
MASA & & & & & & & &
\end{tabular}

El tercer óxido en orden de contenidos es el $\mathrm{Fe}_{2} \mathrm{O}_{3}$, lo cual se puede ser relacionado al encontrar partículas magnéticas en las arenas. Es bien sabido que el óxido de titanio es una impureza que está presente en el caolín, lo cual se muestra en la tabla referida. Es notoria la cantidad de óxido de hierro y sodio, lo cual puede ser atribuido a los feldespatos encontrados en DRX, como las alunitas y ortoclasas.

\subsection{Análisis cristalográfico}

La figura 1 muestra el difractograma de la arena de caolín, en donde se pueden observar las fases cristalinas presentes en el mineral.

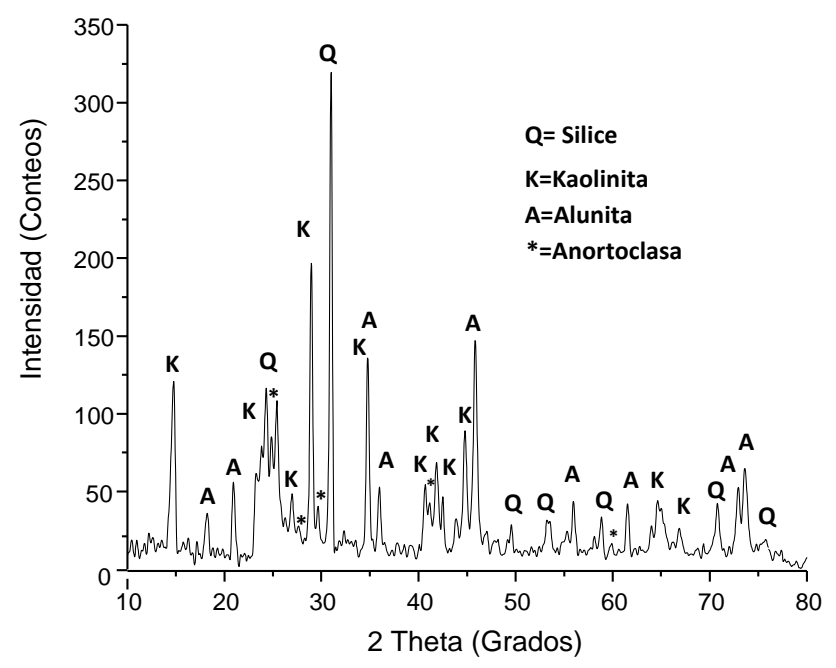

Figura 1. Difractograma de la arena de caolín. 
Puede apreciarse que las señales más importantes son de cuarzo $\mathrm{SiO}_{2}$, caolinita $2 \mathrm{SiO}_{2} \cdot \mathrm{Al}_{2} \mathrm{O}_{3} \cdot 2 \mathrm{H}_{2} \mathrm{O}$ y alunita $\mathrm{KAl}_{3}\left(\mathrm{SO}_{4}\right)_{2}(\mathrm{OH})_{6}$. Estos resultados son acordes al análisis químico de la tabla 1. También están presentes otros cristales en menores proporciones tales como la Anorthoclasa (K,Na) $\mathrm{AlSi}_{3} \mathrm{O}_{8}$ con hematita $\left(\mathrm{Fe}_{2} \mathrm{O}_{3}\right)$ y magnentita $\left(\mathrm{Fe}_{3} \mathrm{O}_{4}\right)$. Cabe mencionar que existe la posibilidad de encontrar otras impurezas como los oxihidróxidos de hierro $(\mathrm{FeOOH})$, pirita $\left(\mathrm{Fe}_{2} \mathrm{~S}\right)$ y carbonatos de Calcio $\left(\mathrm{CaCO}_{3}\right)$ e ilmenita $\left(\mathrm{Fe}_{1.35} \mathrm{Ti}_{0.65} \mathrm{O}_{3}\right)$, que sin embargo dados sus bajos contenidos inferiores al $0.5 \%$ difícilmente pueden ser detectados por difracción de rayos $\mathrm{X}$.

\subsection{Análisis de la distribución del tamaño de las partículas.}

El estudio de distribución de tamaño de partículas se muestra en la Tabla 2. De los resultados obtenidos del porcentaje en masa, se pone en evidencia $92.1 \%$ de la masa de las partículas posee un tamaño inferior a los $125 \mu \mathrm{m}$, y que el $55.8 \%$ es inferior a $38 \mu \mathrm{m}$, (malla 400). Se calculó el tamaño promedio estadístico obteniendo un resultado de $46.34 \mu \mathrm{m}$. Este resultado no proporciona una información precisa debido no se cuenta con tamices muy finos, puesto que el porcentaje en masa es de $55.8 \%$, se tomó la decisión de hacer un análisis de las partículas finas por medio de un equipo de difracción láser.

Tabla 2. Distribución de tamaños de partículas en húmedo por mallas.

\begin{tabular}{cccc}
\hline \#Malla & Promedio Abertura & \% Masa & Acumulativo de finos \\
\hline+30 & $600 \mu \mathrm{m}$ & 0 & 100 \\
$-30+50$ & $600 \mu \mathrm{m}$ a $300 \mu \mathrm{m}$ & 0.4 & 99.6 \\
$-50+80$ & $300 \mu \mathrm{m}$ a $190 \mu \mathrm{m}$ & 1.4 & 98.2 \\
$-80+100$ & $190 \mu \mathrm{m}$ a $149 \mu \mathrm{m}$ & 1.2 & 97 \\
$-100+120$ & $149 \mu \mathrm{m}$ a $125 \mu \mathrm{m}$ & 2.8 & 94.2 \\
$-120+150$ & $125 \mu \mathrm{m}$ a $105 \mu \mathrm{m}$ & 2.1 & 92.1 \\
$-150+200$ & $105 \mu \mathrm{m}$ a $74 \mu \mathrm{m}$ & 7.5 & 84.6 \\
$-200+325$ & $74 \mu \mathrm{m}$ a $45 \mu \mathrm{m}$ & 19.9 & 64.7 \\
$-325+400$ & $45 \mu \mathrm{m}$ a $38 \mu \mathrm{m}$ & 8.9 & 55.8 \\
-400 & $38 \mu \mathrm{m}$ & 55.8 & 0 \\
\hline
\end{tabular}

Como se mencionó, la finalidad de analizar la distribución de tamaños de las partículas más finas, que no es posible determinarlo con las mallas de laboratorio, se realizó un segundo estudio de distribución de tamaños por medio de un analizador por difracción láser; el cual es mostrado en la figura 2a) y b).
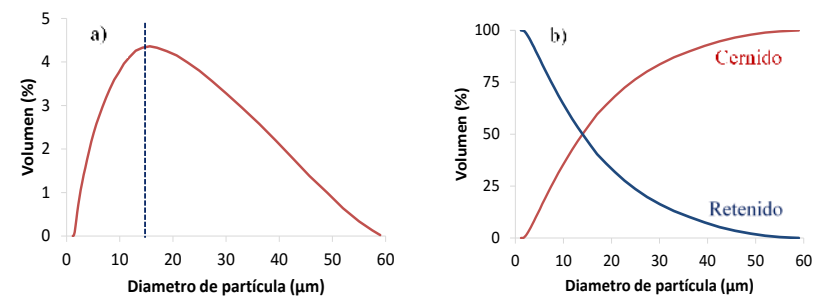

Figura 2. Distribución de tamaños de partícula determinado por difracción láser del material fino que pasa por la malla $400(38 \mu \mathrm{m})$. a) Distribución del diámetro de la partícula y $\%$ volumen y b) Cernido y retenido.
La gráfica 2a), presenta partículas con una curva que exhibe un punto máximo que representa el tamaño promedio de la partícula, en aproximadamente $14 \mu \mathrm{m}$, (se señala con una línea punteada). Sin embargo en la gráfica, también se logra apreciar que el área bajo la curva de las partículas de este tamaño, y hacia las más finas, ocupa una pequeña parte del área total. Lo anterior sugiere un estudio más profundo de estas partículas finas, en el cual se determine la viabilidad de que éstas pudieran retornar al proceso de la planta caolín. En la figura $2 \mathrm{~b}$ ) se observa prácticamente el mismo valor al $50 \%$ del volumen de partículas, el cual se presenta al graficar las curvas de retenido y cernido, cuyo punto de intersección es precisamente a $14 \mu \mathrm{m}$.

\subsection{Estudio de Microscopía Óptica}

El estudio realizado de las muestras obtenidas por microscopia óptica, para los productos a $-30+50 \#(600 \mu \mathrm{m}$ a $300 \mu \mathrm{m})$, se muestran en la figura 3. Cabe mencionar que se observaron todos los rangos de mallas, observándose la misma distribución de colores de partículas en todas las muestras analizadas. Se observan principalmente cuatro grupos de partículas: a) transparentes, b) color blanco c) color rojo naranja, y d) color gris - negro. La fase cristalina que más predomina es el cuarzo, lo cual está en acuerdo con los análisis químicos y cristalográficos, además se pone en evidencia que en el proceso realizado en la planta piloto, se retira principalmente sílice [8]. El color transparente puede ser atribuido al cuarzo, encontrado en DRX, como fase cristalina mayoritaria. El color rojo - naranja puede ser atribuido a la presencia de los óxidos de hierro, probablemente unidos a los alumnosilicatos. Finalmente el color gris negro pueden ser atribudos a la alunita y óxidos de hierro.

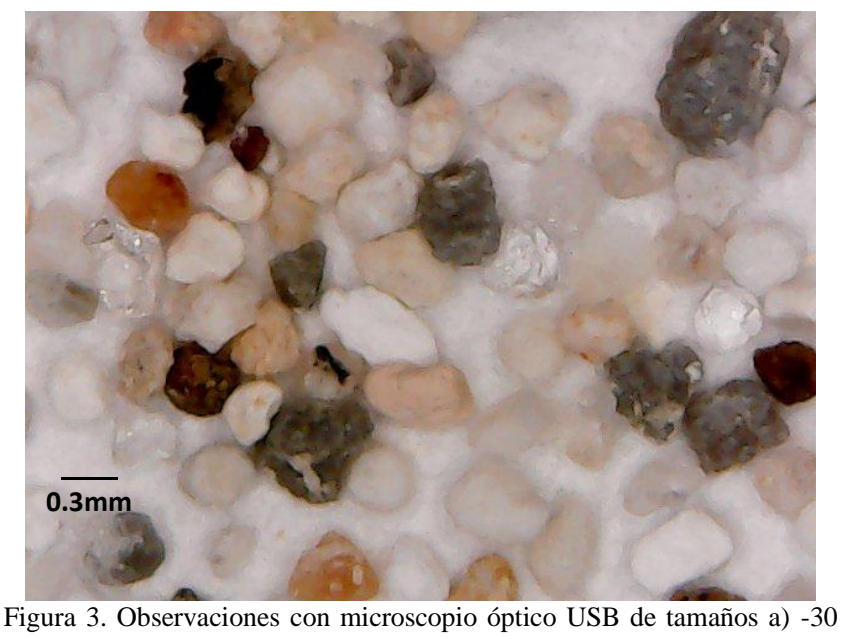
+50 \# $(600 \mu \mathrm{m}$ a $300 \mu \mathrm{m})$.

En la tabla 3 se observan las características de las arenas. Nótese un tamaño promedio de todas las partículas (global) de $46.34 \mu \mathrm{m}$. Es un tamaño promedio fino, debido a que más del $50 \%$ de las partículas son inferiores a $38 \mu \mathrm{m}$. Sin embargo al analizar las partículas finas, el área bajo la curva indica que la cantidad de partículas inferiores al tamaño promedio a $14 \mu \mathrm{m}$ es bajo. 
Tabla 3. Características físicas de las arenas de caolín.

\begin{tabular}{ccccc}
\hline $\begin{array}{c}\text { Tamaño promedio } \\
\text { de todas las } \\
\text { partículas }(\mu \mathrm{m})\end{array}$ & $\begin{array}{c}\text { Tamaño promedio } \\
\text { de las partículas } \\
\text { finas }(\mu \mathrm{m})\end{array}$ & $\begin{array}{c}\text { Densidad } \\
\left(\mathrm{g} / \mathrm{cm}^{3}\right)\end{array}$ & $\begin{array}{c}\text { Magnéticos } \\
\text { Tubo Davis } \\
(\%)\end{array}$ & $\begin{array}{c}\text { Magnéticos } \\
(\%)\end{array}$ \\
\hline 46.34 & 14 & 2.56 & 6.64 & 10.43 \\
\hline
\end{tabular}

Otro dato que llama la atención es el porcentaje de partículas magnéticas. Con estos resultados puede deducirse que el tubo Davis, a pesar de ser un equipo costoso y cuyo empleo requiere de un importante gasto de energía eléctrica, llegó ser obsoleto comparado con un imán de neodimio, el cual se puede encontrar en tiendas comerciales y se emplea sin costo energético. Además empleando el imán de neodimio-fierroboro la captación de partículas magnéticas fue $3.79 \%$ mayor. Este resultado sugiere analizar este tipo de partículas magnéticas cuyo contenido metálico pudiera ser económicamente interesante.

\section{Conclusión}

El residuo de caolín estudiado, posee un bajo porcentaje de alúmina, por lo que convendría mezclarlo con otros materiales como la alúmina para inferir una aplicación refractaria e incrementar sus propiedades mecánicas. Contiene una cantidad importante de feldespatos, así como de sílice y de partículas magnéticas, estas últimas con un porcentaje importante de $10.43 \%$ en masa, podrían ser separadas y obtener un subproducto el cual sería interesante investigar. Se encontró un tamaño promedio de 14 micrómetros y el $55.8 \%$ en masa es de un tamaño inferior a $38 \mu \mathrm{m}$, éste tipo de partículas debe ser analizado para determinar la posibilidad de retornarlas a la planta de caolín y disminuir la cantidad de residuos finos generados. El tubo Davis de laboratorio, a pesar de ser un equipo costoso ahora ya es obsoleto comparado con un imán de neodimio. Finalmente se sugiere hacer un estudio exhaustivo de este material a fin de analizar su reactividad térmica y química.

\section{English Summary}

\section{Characterization of kaolin sands processing.}

\section{Abstract}

Sands from the processing of kaolin are characterized exhaustively. A crystallographic analysis by X-ray diffraction, analysis of the particle size distribution by sieving, as well as by laser diffraction; moisture, density, percentage of magnetic material and morphology were determined. The crystallographic studies show that the predominant phases are silica, kaolinite, anorthoclase and alunite. An average particle size of $46.34 \mu \mathrm{m}$ was found. Optical microscopy images show different types of particles of different shades corresponding to polymorphic quartz, silicoaluminates and feldspars.

Keywords: Kaolin sands, characterization, magnetism.

\section{Agradecimientos}

Los autores agradecen la Química Martha López Tolentino, por su amable colaboración para el desarrollo de éste trabajo.

\section{Referencias}

Legorreta-García, F, B., 2013. Estudio de la Remoción de Impurezas de Arcillas Caoliníticas del Estado de Hidalgo (México). Revista Latinoamericana de Metalurgia y Materiales, RLMM, 33(2).

Okada, K., Toya, T., Kameshima, Y., \& Nakajima, A. 2004. WIT Transactions on Ecology and the Environment. Properties of glassceramics prepared from Kira (waste by-products of silica sand and kaolin clay refining) and various additives, pp. 79 .

Menezes, R. R., Farias, F. F., Oliveira, M. F., Santana, L. N., Neves, G. A., Lira, H. L., \& Ferreira, H. C. 2009. Waste Management \& Research. Kaolin processing waste applied in the manufacturing of ceramic tiles and mullite bodies, 27(1), pp. 78-86.

Menezes, R. R., Brasileiro, M. I., Santana, L. N., Neves, G. A., Lira, H. L., \& Ferreira, H. C. 2008. Waste Management \& Research. Utilization of kaolin processing waste for the production of porous ceramic bodies., 26(4), pp. 362-368.

Menezes, R. R., Brasileiro, M. I., Gonçalves, W. P., Santana, L. N. D. L., Neves, G. A., Ferreira, H. S., \& Ferreira, H. C. 2009. Materials Research. Statistical design for recycling kaolin processing waste in the manufacturing of mullite-based ceramics. 12(2), pp. 201-209.

Toya, T., Tamura, Y., Kameshima, Y., \& Okada, K. (2004). Ceramics international. Preparation and properties of $\mathrm{CaO}-\mathrm{MgO}-\mathrm{Al}_{2} \mathrm{O}_{3}-\mathrm{SiO}_{2}$ glass-ceramics from kaolin clay refining waste (Kira) and dolomite. 30(6), pp. 983-989.

Kobayashi, Y., Sumi, K., \& Kato, E. 2000. Ceramics International. Preparation of dense cordierite ceramics from magnesium compounds and kaolinite without additives. 26(7), pp. 739-743.

Azeredo, G., \& Diniz, M. 2013. Construction and Building Materials. Self-compacting concrete obtained by the use of kaolin wastes. 38, pp. 515-523.

Gomes, K. C., Rocha, B. D., Ferreira, D. T., de Lira, E. C., Torres, S. M., de Barros, S. R., \& Barbosa, N. P. 2012. Trans Tech Publications. Activation Alkaline Waste Kaolin for Fabrication of Building Blocks. In Key Engineering Materials Vol. 517, pp. 622-627.

Maia, A. A. B., Angélica, R. S., \& Neves, R. F. 2011. Clay Minerals. Use of industrial kaolin waste from the Brazilian Amazon region for synthesis of zeolite A., 46(1), pp. 127-136.

Hildebrando, E. A., Andrade, C. G. B., Rocha Junior, C. A. F. D., Angélica, R. S., Valenzuela-Diaz, F. R., \& Neves, R. D. F. (2014). Materials Research. Synthesis and characterization of zeolite NaP using kaolin waste as a source of silicon and aluminum. 17, pp. 174179. 